Nouvelles perspectives en sciences sociales

Revue internationale de systémique complexe et d'études relationnelles

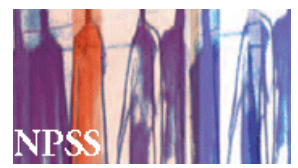

\title{
Pouvoir et dynamique de groupe
}

\section{Mélanie Girard}

Volume 8, numéro 2, mai 2013

URI : https://id.erudit.org/iderudit/1016475ar

DOI : https://doi.org/10.7202/1016475ar

Aller au sommaire du numéro

\section{Éditeur(s)}

Prise de parole

\section{ISSN}

1712-8307 (imprimé)

1918-7475 (numérique)

Découvrir la revue

\section{Citer cet article}

Girard, M. (2013). Pouvoir et dynamique de groupe. Nouvelles perspectives en sciences sociales, 8(2), 177-208. https://doi.org/10.7202/1016475ar

\section{Résumé de l'article}

La phénoménologie a beaucoup insisté sur la liberté de l'acteur social. Cette approche et celles qui en découlent connaissent des limites importantes. La pleine reconnaissance de ces limites suppose un déplacement paradigmatique de l'action vers la relation. Ce déplacement suppose une remise en question de la notion de pouvoir telle qu'elle est modélisée par la phénoménologie et ses dérivés, que l'on peut qualifier de perspectives surrationnalisantes. Dans cette perspective, la plupart des théories présentent le pouvoir comme une chose détenue; une approche relationnelle le présente comme une catégorie analytique non utile. Le travail que voici rend compte d'une démarche d'opérationnalisation du pouvoir comme détenu, lequel renvoie à une distribution formelle de l'autorité. Elle le fait en créant des indicateurs qui sont soumis à l'empirie. Elle conclut à la non-détermination du pouvoir formel dans la circulation de l'information et ouvre ainsi la voie à des modélisations dans lesquelles le pouvoir n’apparaît plus comme un concept central pour la compréhension des relations humaines. 


\section{Pouvoir et dynamique de groupe}

\section{MÉlanie GiRARD}

Université de Hearst

e texte rend compte d'une démarche d'opérationnalisation
du concept de pouvoir tel qu'il a été et tel qu'il est toujours principalement utilisé en sciences sociales. L'analyse porte sur des réunions filmées en France. Elle se fait à partir d'indicateurs qui se dégagent des conclusions que l'on tire des modélisations que proposent, depuis maintenant plus de deux siècles, les spécialistes des sciences sociales qui conçoivent le pouvoir comme une chose que l'on détient. Dans une première partie, nous posons les principes de l'approche relationnelle à partir de laquelle nous proposons d'étudier les relations humaines afin de sortir de l'impasse dans laquelle place une perspective phénoménologique, axée sur l'acteur, sur son pouvoir et sur son intérêt. Dans une deuxième partie, nous présentons le lien entre pouvoir et intérêt. Dans une troisième partie, nous distinguons entre pouvoir théorique et pouvoir effectif ou informel. Les indicateurs du pouvoir formel et les hypothèses qui s'y rattachent sont présentés dans une quatrième partie. Dans une cinquième partie, nous nous penchons sur l'échantillon, l'instrument de collecte des données et le déroulement de cette collecte. Une sixième partie est réservée à la description des résultats obtenus à partir des analyses effectuées. Dans une septième partie, nous procédons à l'inter- 
prétation de ces résultats, pour conclure sur la démarche dans une huitième et dernière partie.

Les sciences sociales se sont largement construites sur l'idée d'un acteur rationnel. Ce fait n'est plus à démontrer. Le principe d'un acteur rationnel - et donc conscient, stratégique, intentionnel, libre, réfléchi, intéressé - a donné cours à de nombreuses modélisations qui n'ont su rendre compte de toute la complexité de l'action humaine; qui ont réduit l'humain à sa seule conscience. Plusieurs, cependant, ont fait entendre des critiques. De l'antiutilitarisme $e^{1}$ à l'infra-intentionnalisme ${ }^{2}$, on a revendiqué une vision plus théoriquement juste de l'action. De la systémique ${ }^{3}$ aux études relationnelles ${ }^{4}$, en passant par la cybernétique ${ }^{5}$ et la complexité ${ }^{6}$, on a insisté sur l'importance de la dialectique - la

$1 \quad$ Alain Caillé, Critique de la raison utilitaire, Paris, La Découverte, 1989; Alain Caillé, et Jacques T. Godbout, L'Esprit du don, Paris, La Découverte, coll. "Textes à l'appui / Textes anthropologiques », 1992.

2 Paul Ladrière, Patrick Pharo et Louis Quéré, La Théorie de l'action. Le sujet pratique en débat, Paris, CNRS, 1993.

3 Pascal Roggero, De la complexité des politiques locales, Paris, L'Harmattan, 2005; Claude Vautier, Le Repli et l'imagination. Essai de modélisation complexe des phénomènes territoriaux: le cas de l'intercommunalité dans le département du Tarn, thèse de doctorat, Toulouse, Université des Sciences Sociales de Toulouse I, 2005.

4 Rachid Bagaoui, "Un Paradigme systémique relationnel est-il possible? Proposition d'une typologie relationnelle ", Nouvelles Perspectives en sciences sociales, vol. 3, nº 1, 2007, p. 151-175; Mélanie Girard, "Éléments de critique des théories de l'action ", Nouvelles Perspectives en sciences sociales, vol. 3, n `1, 2007, p. 47-60; Mélanie Girard, Contribution à la critique des théories de l'action. Intention et émoraison, thèse de doctorat, Toulouse, Université des Sciences Sociales de Toulouse I, 2009; Mélanie Girard, Simon Laflamme et Pascal Roggero, "LIntention est-elle si universelle que ne le prétendent les théories de l'action? ", Nouvelles Perspectives en sciences sociales, vol. 1, $\mathrm{n}^{\circ} 2$, 2006, p. 115-148; Simon Laflamme, Communication et émotion. Un essai de microsociologie relationnelle, Paris, L'Harmattan, coll. "Logiques sociales ", 1995; Simon Laflamme, Suites sociologiques, Sudbury, Prise de parole, coll. "Épistémè », 2006; Claude Vautier, "Essai de modélisation complexe en sciences sociales : le cas de l'intercommunalité dans le département du Tarn ", Nouvelles Perspectives en Sciences Sociales, vol. 1, no 2, 2006, p. 149-217; Claude Vautier, «La Longue marche de la sociologie relationnelle ", Nouvelles perspectives en sciences sociales, vol. 4, n 1, 2008, p. 77-106.

5 Heinz von Foerster, Observing Systems: Selected Papers of Heinz von Foerster, Seaside (CA), Intersystems Publications, 1981.

6 Edgar Morin, Introduction à la pensée complexe, Paris, ESF, 1992. 
dialogie chez Morin ${ }^{7}$-, de la relation. Et c'est ainsi que les sciences sociales sont devenues de plus en plus suspicieuses à l'égard des modélisations à caractère surrationnalisant, qui placent l'intention, l'intérêt ou la stratégie par devant même des relations.

\section{Les principes de l'approche relationnelle}

La relationalité, en sciences sociales, est relativement nouvelle. Comme la complexité, elle souffre parfois d'abus de sens, d'économie de latitude dans la conceptualisation qu'elle inspire. La relation entre interlocuteurs est en fait à la relationalité ce que la complication est à la complexité : si l'on considère souvent, à tort, que ce qui est compliqué est nécessairement complexe, on estime souvent que la notion de relation renvoie strictement à des formes micrologiques de la socialité. Il faut dire que l'interactionnisme a bien aménagé la position, de Mead à Goffman, en rappelant que l'humain se construit dans l'interaction; et que l'ethnométhodologie et l'individualisme méthodologique ont rappelé les limites de l'individu en insistant sur le fait que, bien que libre et rationnel, l'humain est toujours inscrit dans des interactions, ou des relations interpersonnelles, qui régissent ses modes d'action. Mais ce que ces sociologies ont oublié, c'est que la relationalité est aussi d'ordre macrologique; que si les humains sont toujours en relation, c'est, certes, parce que la vie sociale oblige à l'interaction, mais c'est d'abord et avant tout parce que l'humanité est, par son essence même, relationalité. Si l'identité se construit toujours dans un rapport à l'autre, comme l'ont bien établi les interactionnistes, c'est que les êtres qui la portent, la construisent, la vivent, la subissent, sont toujours en relation. Et que ces êtres-en-relation - dans la mesure où la relation suppose un rapport au langage - s'inscrivent dans des codes, dans des symboliques qui les dépassent dans et par lesquelles ils se distinguent et se retrouvent ou se reconnaissent dans leur similitude. Se distinguent parce que le langage, en tant qu'il est

\section{Ibid.}


lieu d'émoraison ${ }^{8}$, permet l'expression de l'être, de l'unicité, c'est-à-dire de la façon particulière dont l'individu " filtre " les codes en fonction de son historicité et de sa socialité. Se retrouvent et se reconnaissent dans leur similitude parce que le langage, en tant qu'il est lieu d'émoraison, est aussi, justement, celui de la récurrence, c'est-à-dire de la création de valeurs communes, de modes de pensée semblables, d'émotions comparables... Une approche relationnelle tient compte de cela dans ses modélisations; comme le font les approches systémiques; comme le font les approches qui se réclament de la complexité. Reconnaître le caractère relationnel de l'action humaine, c'est reconnaitre que la relation est le propre de l'humain au sens où l'humain ne peut être qu'en relation, au sens où il est intrinsèquement lié, rattaché au monde qui l'entoure; c'est voir dans le langage, dans la communication humaine, l'expression de la nécessaire mise en commun des symboliques, du vécu, de l'émotion, de l'histoire, de la raison...; c'est comprendre que, sans même avoir échangé des mots, des regards, des touchers, les êtres sont en relation parce qu'ils existent relativement à un autre, toujours - sans vouloir ici donner dans un excès de relativisme -, parce qu'ils construisent collectivement les symboliques en existant, en émettant des opinions, en travaillant, en consommant, en aimant, en votant, en enseignant... et en inscrivant ces actions dans des modes singuliers, mais forcément récurrents.

La démarche que voici, en tant qu'elle s'inscrit dans une approche relationnelle, modélise et opérationnalise en tenant compte de ce que l'humain est un être social, communicationnel, historique, conscient et inconscient, rationnel et irrationnel, intéressé et non intéressé... Cela suppose un déplacement paradigmatique, de l'action vers la relation. Un tel déplacement sous-tend une critique des théories de l'action, des théories au caractère surrationnalisant dans lesquelles l'acteur occupe toujours la place centrale. Cette critique oblige à soumettre la modélisation surrationnalisante à l'empirie en manipulant, dans les exercices respectifs que suppose cette confrontation, autant

\footnotetext{
8 Simon Laflamme, Communication et émotion..., op. cit.
} 
de variables que l'impose la critique; ainsi pourra-t-on connaître la réelle valeur heuristique de la modélisation, c'est-à-dire sa capacité à rendre compte de l'action sociale, ou, pour le dire autrement, à modéliser adéquatement son objet.

\section{Le lien entre pouvoir et intérêt}

Les modélisations à caractère rationalisant investissent leur objet, l'humain, d'une grande autonomie. Cette autonomie découle de la capacité qu'a l'humain d'agir en fonction de son intérêt; la conscience qu'il a de son intérêt lui permet de développer des stratégies, en lien avec ses intentions... tout cela, parce qu'il est rationnel. Les catégories s'interpellent mutuellement ${ }^{9}$. Et elles appellent une conception particulière du pouvoir.

Un premier travail ${ }^{10}$ a été consacré à la création et l'opérationalisation d'indicateurs liés à une modélisation surrationnalisante, d'une part, et à une modélisation relationnelle, de l'autre. Le travail en question visait à mettre à l'épreuve, par le biais de l'étude des propos et des actions de membres de rassemblements divers (assemblées délibératives, comités, associations...), les principes des théories de l'action, selon lesquels les individus agissent davantage en fonction de leur intérêt personnel - donc en mettant en œuvre des intentions à l'aide de stratégies particulières - qu'ils ne sont déterminés par les dynamiques dont ils participent, sans lesquelles ne peuvent se livrer intérêt, intention et stratégie, lesquelles possibilités d'action dépendent, de toute façon, de la socialité, de l'historicité et de l'émoraison qui façonnent les relations humaines. Ce travail portait sur des échanges ponctuels et ne permettait pas d'observer l'évolution des relations; ce faisant, il ne s'intéressait pas directement aux structures de pouvoir. Or, le pouvoir constitue une catégorie analytique privilégiée des théories de l'action. Car si l'humain peut faire agir en fonction de son intérêt, il le peut d'autant plus, selon elles, qu'il est doté de pouvoir. L'intérêt, tel qu'il est

\footnotetext{
9 Mélanie Girard, "Éléments de critique des théories de l'action ", op. cit.

10 Mélanie Girard, Contribution à la critique des théories de l'action. Intention et émoraison, op. cit.
} 
modélisé par les théories de l'action, a pour corollaire le pouvoir dans le sens où sont théoriquement plus à même de faire valoir leurs intérêts les individus dont la marge de pouvoir formel est grande; et ce pouvoir apparaît dès lors comme détenu.

Il existe, en fait, en sciences sociales, trois théories du pouvoir : celle qui présente le pouvoir comme détenu, celle qui conçoit le pouvoir de façon relationnelle, celle qui estime que le pouvoir est une catégorie non analytiquement utile.

La première est le propre des théories où l'on centre tout sur l'acteur. Depuis Weber ${ }^{11}$ surtout, ces théories insistent sur l'idée selon laquelle le pouvoir constitue un instrument dont dispose l'acteur, qui lui permet d'agir sur le monde en faisant faire aux autres malgré leur volonté.

Dans la seconde de ces théories, le pouvoir est compris comme relationnel; cette conception du pouvoir est encore le propre des théories de l'acteur, mais elle appartient aussi aux théories des systèmes. Crozier et Friedberg ${ }^{12}$ et Friedberg lui-même ${ }^{13}$, par exemple, présentent le pouvoir comme relationnel, bien qu'en confondant deux registres : le pouvoir est « une relation et non un attribut des acteurs ${ }^{14}$, donc il se livre dans la dynamique et dépend d'elle; cependant, il est également détenu par l'acteur, qui l'exerce tout en tentant de maîtriser les zones d'incertitude.

La troisième théorie du pouvoir est celle où le pouvoir apparaît comme catégorie non analytiquement utile; on insiste alors sur la relation, c'est-à-dire sur ce qui fait qu'il y a du lien social, de la socialité plutôt que de l'action. Dans une telle optique, la notion de pouvoir s'avère peu utile pour expliquer la dynamique, parce que, s'il y a pouvoir, l'ensemble de ce qui advient n'est pas attribuable au pouvoir ${ }^{15}$; du moins, pas au pouvoir que l'on peut

$11 \quad$ Max Weber, Économie et société, Paris, Plon, coll. " Recherches en sciences humaines ", 1971 [1922].

12 Michel Crozier et Erhard Friedberg, L'Acteur et le système : les contraintes de l'action collective, Paris, Seuil, coll. "Sociologie politique ", 1981 [1977].

13 Erhard Friedberg, Le Pouvoir et la Règle, Paris, Seuil, coll. "Sociologie », 1993.

14 Michel Crozier et Erhard Friedberg, L'Acteur et le système, op. cit., p. 65

15 Voir, sur le plan théorique, Simon Laflamme, La Société intégrée. De la circulation des biens, des idées et des personnes, New York / Bern / Paris, Peter Lang, 1992; Simon Laflamme, Suites sociologiques, op. cit. Voir, sur le plan 
qualifier de formel, ce sur quoi nous reviendrons sous peu. Il devient alors important de comprendre les éléments qui composent la dynamique en tant qu'elle est le lieu de rapports de pouvoir, certes, mais surtout, en tant qu'elle est le lieu où s'expriment la socialité, l'historicité, l'émotion et la raison, lesquelles ne sauraient être réduites à la seule catégorie " pouvoir " lorsque celle-ci sous-tend une distribution formelle de l'autorité. Dans une modélisation relationnelle telle que celle qui est proposée ici, le pouvoir ainsi modélisé ne peut pas constituer une catégorie essentielle; pas plus que ne le peut l'intention ou l'intérêt ou la stratégie. Chez Habermas ${ }^{16}$, dans plusieurs des conclusions que l'on tire de ses affirmations, on constate qu'il en est de même. On retrouve aussi des indices de cette non-utilité du pouvoir chez Crozier et Friedberg ${ }^{17}$, puis chez Foucault ${ }^{18}$, qui, même s'il dit aussi le contraire, rappelle que le pouvoir est partout, qu'il est pluriel et multiple, qu'il est non pyramidal, même lorsque la structure par laquelle il se manifeste est, elle, à l'image d'une pyramide. Tout ce qui est inégalité n'est pas pouvoir; exister dans des rapports d'inégalité, ce n'est pas être dominé, ce n'est pas faire en sorte que tout ce qui advient soit le résultat de cette inégalité. Si Foucault a permis de comprendre cela, il faut dire que, déjà chez Hegel, l'analogie du maître et de l'esclave aménageait bien le terrain en rappelant à quel point le pouvoir est forcément relationnel, mais surtout, dialectique. Plusieurs ont senti ce trop grand réductionnisme et insistent sur la nécessaire distinction entre pouvoir formel et pouvoir effectif; mais ne réaffirment-ils

empirique, Mélanie Girard, Relations humaines et production d'information : l'échange comme objet d'étude d'une approche relationnelle, mémoire de maîtrise, Sudbury, Université Laurentienne, 2004; Mélanie Girard, Contribution à la critique des théories de l'action... op. cit.; Mélanie Girard, Simon Laflamme et Pascal Roggero, "L'Intention est-elle si universelle que ne le prétendent les théories de l'action ?", op. cit.; Paul Jalbert, "Analyse du rôle de l'intention dans les échanges dyadiques ", Nouvelles Perspectives en sciences sociales, vol. 2, $\mathrm{n}^{\circ}$ 1, 2006, p. 101-141.

16 Jürgen Habermas, Théorie de l'agir communicationnel, tomes I et II, Paris, Fayard, coll. "L'espace du politique », 1987 [1981].

17 Michel Crozier et Erhard Friedberg, L'Acteur et le système, op. cit.

18 Michel Foucault, Histoire de la sexualité I. La volonté de savoir, Paris, Gallimard, coll. «Tel », 1994 [1976]. 
pas alors, tout simplement, l'importance de la dynamique en soutenant que ce qui advient n'est pas attribuable à la distribution formelle de l'autorité? N'était-ce là, en fait, l'une des conséquences qu'il faut tirer des conclusions de Crozier et Friedberg, qui constatent l'existence d'un pouvoir que l'on pourrait qualifier d'informel et qui est, au fond, positionnel ou situationnel?

\section{Pouvoir théorique et pouvoir effectif ou informel}

Le pouvoir est ici défini et conceptualisé dans sa forme théorique, c'est-à-dire suivant une distribution formelle de l'autorité, une représentation conceptuelle, un organigramme; il est ainsi compris comme corollaire de l'autorité formelle. Certains rappellent, nous l'avons dit, que distribution formelle de l'autorité et effectivité du pouvoir - ou pouvoir formel et informel - représentent parfois des réalités bien différentes; notre travail permet d'établir dans quelle mesure il y a correspondance entre l'une et l'autre. À partir de l'étude d'une distribution formelle de l'autorité, donc de l'analyse des gestes et des propos émis par chaque membre du groupe, en tenant compte des positions théoriques de pouvoir et de non-pouvoir de chacun, nous mesurons, en fait, le pouvoir " effectif » ou informel, le pouvoir étant ici conçu comme empiriquement observable, mesurable, à l'aide d'indicateurs que nous créons à partir des principes que retiennent les adeptes de la vision du pouvoir comme chose détenue. Si l'autorité formelle confère un pouvoir réel, l'analyse saura le révéler; sinon, ce sera que tout le monde a du pouvoir, que le pouvoir provient de partout, qu'il n'est pas pyramidal et qu'il est, en cela, inhérent aux relations humaines. Ce sera alors que le pouvoir informel a préséance sur le pouvoir formel. On comprendra ainsi que, dans la mesure où tout le monde a du pouvoir, il est moins utile de chercher à faire la preuve de son existence que de découvrir comment il se livre dans et par les relations, dans son informalité. Et si l'on constate que le pouvoir est lié à la détention d'information, à la capacité de compréhension, à l'aptitude à l'écoute, à la capacité de s'exprimer, à la sensibilité aux autres, on aura compris qu'il est plus informel que formel; et que c'est donc 
beaucoup plus la dynamique à l'œuvre que ce n'est le pouvoir formel lui-même qui permet de comprendre ce qui advient dans un échange donné, si inégalitaire soit-il. Car la sensibilité, la capacité de rendre et de s'exprimer, l'écoute, l'intégration sont toutes des facultés que l'être social acquiert et développe en relation, dans des dynamiques, grâce à son émoraison et au caractère émorationnel des relations. Si c'est en effet ce que nous observons, alors nous aurons découvert que la relation, qui est elle-même génératrice de socialité, d'historicité et d'émoraison en même temps qu'elle est générée et entretenue par elles, est plus apte à fournir des éléments de compréhension de l'action sociale que ne l'est une conception phénoménologique du pouvoir.

\section{Hypothèses et indicateurs}

Pour cette modélisation du pouvoir qui est la nôtre, nous avons opté pour la démarche suivante : dégager les principes soutenus par la " théorie du pouvoir comme détenu »; modéliser le pouvoir en fonction d'eux en nous dotant d'indicateurs; soumettre les indicateurs à l'empirie en nous fondant sur cinq verbatim tirés de réunions filmées, au sein d'un même groupe, sur une période de huit mois; dégager les résultats d'une série de fréquences, d'analyses de corrélation et d'analyses de variance à un facteur; conclure sur la valeur heuristique de la catégorie " pouvoir " pour l'analyse de la communication dans les groupes; conclure sur l'importance de l'intérêt personnel pour l'analyse de la communication dans les groupes. Dans la mesure où ces opérations révéleraient le caractère peu utile de la catégorie analytique " pouvoir ", nous estimons, compte tenu de ce que plusieurs travaux ont déjà démontré ${ }^{19}$, qu'il sera légitime de proposer, une nouvelle fois, de comprendre les relations humaines d'un point de vue relationnel en faisant appel aux concepts d'historicité, de socialité et d'émoraison.

19 Pierre Bouchard, "La Théorie de l'action et les parcours de vie ", Nouvelles Perspectives en sciences sociales, vol. 1, n 2, 2006, p. 67-114; Mélanie Girard, Relations humaines et production d'information... op. cit.; Mélanie Girard, Contribution à la critique des théories de l'action... op. cit.; 2009; Paul Jalbert, "Analyse du rôle de l'intention dans les échanges dyadiques ", op. cit. 
De Weber à Habermas, mais déjà chez Machiavel $^{20}$, si l'on tire toutes les conséquences de ce qui est affirmé, on retient qu'il y a pouvoir :

- s'il y a autorité;

- s'il y a influence;

- si l'on peut faire agir contre la volonté;

- si l'on peut faire agir en fonction de son intérêt;

- si l'on peut faire agir contre l'intérêt de la collectivité.

Dans un groupe donné, les gens ont des positions. Ces positions sont liées, par exemple, à des fonctions, à des obligations, à des titres, à des privilèges. Les intentionnalistes ont tôt fait de voir dans ces positions la manifestation du pouvoir; d'un pouvoir pyramidal, déterminant. Ils ont théorisé ce pouvoir, autour de l'idée d'un acteur intéressé, mais ne l'ont pas observé. S’ils s'étaient adonnés à une telle tâche, ils auraient sans doute soutenu que, s'il y a autorité, influence, capacité à faire agir indépendamment de la volonté, en fonction de son intérêt et contre l'intérêt de la collectivité, on devrait normalement :

- observer, dans des échanges, des gens ou des groupes qui ont du pouvoir;

- observer, dans des échanges, des gens ou des groupes qui n'ont pas de pouvoir;

- dégager une structure qui rend compte de ce que certains détiennent plus de pouvoir que d'autres;

- repérer une évolution du propos, dans le temps, en fonction des positions de pouvoir;

- observer une manifestation, dans cette évolution, de l'intérêt de l'acteur concerné à consolider son pouvoir, dans la mesure où son pouvoir lui provient d'une position.

C'est ce que nous faisons ici. En nous consacrant à l'étude d'un même groupe dans le temps, nous souhaitons faire écho aux

$\overline{20} \quad$ Nicolas Machiavel, Le Prince, traduit et commenté par Jean-Louis Fournel et Jean-Claude Zancarini, texte italien établi par Giorgio Inglese, Paris, Presses universitaires de France., coll. "Fondement de la politique », 2000 [1532]. 
thèses qui veulent que les jeux de pouvoir s'expriment dans la chronologie; qu'un échange, à lui seul, ne puisse rendre compte de la relation et que c'est grâce à l'étude longitudinale, donc de la relation telle qu'elle se déploie dans le temps, qu'on peut comprendre l'échange en tant que structuré par le pouvoir.

En partant des principes énoncés plus haut, nous avons dégagé une série d'indicateurs à partir desquels nous avons pu construire des questions destinées à l'analyse des données dont nous disposions. Ces indicateurs peuvent être regroupés en trois rubriques: une première qui concerne la prise de parole, une deuxième qui fait appel aux idées, une troisième qui a trait aux alliances. La division sert des fins d'organisation; les frontières entre ces rubriques, il va sans dire, sont perméables, la parole découlant forcément d'idées, les alliances passant par la parole et les idées donnant cours aux alliances qui peuvent se modeler dans un échange donné. Si l'on peut concevoir autrement le pouvoir qui appartiendrait à une distribution formelle de l'autorité et, donc, le mesurer à partir d'indicateurs autres que ceux que nous retenons, on peut aussi admettre que ceux-ci constituent effectivement des indicateurs du pouvoir lorsqu'il est conçu comme détenu.

En ce qui concerne la prise de parole, nous retenons les indicateurs suivants : le nombre de tours de parole, la fréquence des interruptions et la fréquence des oppositions. Nous estimons que si, dans un groupe donné, une personne a de l'autorité ou de l'influence, on devrait pouvoir s'attendre à ce qu'elle prenne la parole plus souvent que ceux qui n'en ont pas, à ce qu'elle soit plus écoutée, à ce qu'elle soit peu interrompue, à ce qu'elle interrompe souvent les autres et à ce qu'on s'oppose peu à ses propos, la menace d'éventuelles représailles s'avérant trop grande.

Pour ce qui est des idées, nous retenons les indicateurs qui suivent : la présentation d'idées ou de thèmes qui ne sont pas à l'ordre du jour; la présentation d'idées nouvelles; la reprise d'idées; le suivi dans les idées, lequel peut s'exprimer par la présence d'un fil conducteur témoignant d'un intérêt ou par la présence de stratégies; les ententes. On peut s'attendre, si la 
théorie du pouvoir en tant que détenu dit vrai, à ce que, dans un groupe donné, une personne qui est en position de pouvoir déroge plus aisément du fonctionnement normatif de la réunion, fonctionnement normalement anticipé par l'ordre du jour. On peut aussi s'attendre à ce qu'elle se sente plus libre de proposer des idées nouvelles, de faire des suggestions; à ce que ses propos et ses idées soient fréquemment repris par d'autres; à ce que les autres membres du groupe changent d'idée en fonction de ses positions et, donc, à ce que les ententes ou les conclusions soient liées à ses positions, à son intérêt; et à ce que cet intérêt soit perceptible dans l'élaboration d'une stratégie, donc d'un événement pluritemporel. On devrait aussi pouvoir s'attendre, de façon générale, à ce que les ententes et les conclusions soient le fruit d'un décret de la part de celui dont la part de pouvoir est la plus grande.

En ce qui a trait aux alliances, nous retenons un indicateur : la manipulation de la part d'individus qui détiennent théoriquement le plus de pouvoir. Nous estimons que, si la théorie dit vrai, dans un groupe donné, les gens qui s'allient le font en tant qu'ils sont manipulés par la personne au pouvoir, qu'ils n’ont pas de position individuelle, indépendante de celle-ci. Et dans cette perspective, il est bien question de " la " personne dont la marge de pouvoir est la plus grande, puisque la logique du pouvoir comme chose détenue, en tant qu'elle suppose la mise en œuvre d'un intérêt personnel - intérêt qui consiste, nous l'avons dit, à consolider la position même par laquelle est conféré à la personne qui en bénéficie le pouvoir dont elle jouit -, occulte l'idée selon laquelle les actes langagiers et paralangagiers par lesquels s'expriment le pouvoir - les interruptions, les oppositions, la présentation d'idées nouvelles ou ne figurant pas à l'ordre du jour, la reprise d'idées, l'élaboration d'un fil conducteur, la mise en œuvre de stratégies, les ententes par décret ou les manipulations visant à créer des alliances - résulteraient d'un effort collectif sans quoi l'intérêt n'est plus, alors, intérêt à proprement parler. Cela dit, afin de donner une chance à la critique, nous nous sommes éloignée, dans l'analyse, d'une logique aussi puriste et avons 
admis la possibilité qu'il existe, chez les gens au pouvoir, un intérêt commun, lié au désir de maintenir ou de consolider leurs positions respectives; et nous avons donc optée, dans l'analyse, pour le repérage de manipulations orientées vers la création d'alliances de la part de tous ceux dont la position de pouvoir était théoriquement élevée.

\section{5. Échantillon, instrument de collecte et déroulement de la collecte}

Les indicateurs ainsi posés, il nous a été possible de formuler des questions qui ont alors constitué la grille d'analyse à laquelle ont été soumises les données issues des cinq rencontres à l'étude. Avant de présenter ces questions, penchons-nous un moment sur l'échantillon.

Le corpus utilisé provient d'une collecte de données effectuée entre les mois de mai et décembre 2005, dans le cadre d'une thèse de doctorat, soit le travail antérieur évoqué plus haut. Les cinq rencontres à l'étude sont celles d'un organisme d'aide humanitaire, situé dans une ville du Sud-Ouest de la France, dont les membres se rencontrent sur une base mensuelle. Les réunions durent en moyenne trois heures et comportent toujours un ordre du jour. Il existe, dans le groupe, des gens qui sont en position théorique de pouvoir - dans la mesure où leur rôle leur confere une certaine autorité et certaines responsabilités - et des gens qui ne le sont pas. Au total, six catégories ou positions sont retenues: " secrétaire du groupe ", " vice-secrétaire du groupe ", " trésorier ", " chargé de dossier de type 1 ", " chargé de dossiers de type 2 ", " sans pouvoir défini ». Le secrétaire du groupe assume le rôle de président de séance; il est chargé de dossiers primaires. Le vice-secrétaire du groupe endosse le rôle de secrétaire en son absence; il est également chargé de dossiers primaires. Le trésorier gère les finances; il est chargé, entre autres choses, de relayer l'information relativement à la cotisation. Les chargés de dossier de type 1 s'occupent de dossiers primaires, mais ne peuvent assumer le rôle du secrétaire en son absence, cette tâche étant réservée au vice-secrétaire; les chargés de dossiers de type 2 sont 
responsables de dossiers secondaires, les dossiers primaires étant jugés plus importants que les dossiers secondaires. Les gens que l'on a qualifiés de "sans pouvoir défini " sont des membres du groupe dont la tâche consiste essentiellement à participer aux réunions.

L'analyse porte surtout sur ce qui est dit. Dans une rencontre donnée, un individu peut s'imposer par le silence et, ainsi, activer, voire asseoir son pouvoir. Cependant, dans la mesure où la parole est le propre de la discussion, de l'argumentation - que cette dernière mène ou non à l'entente - on peut imaginer que l'exercice du pouvoir passe en grande partie par celui de la parole. C'est pourquoi, bien que nous avons tenu compte des gestes manifestes par lesquels s'exprimait une position - se prononce effectivement, par exemple, la personne qui lève la main pour voter -, l'analyse porte pour la plus grande partie sur le verbe, par lequel se livre la raison, l'émotion, la justification, la preuve, la contradiction...

La première des questions qui composent la grille d'analyse consiste à savoir si la personne est ou non en position théorique de pouvoir; la question s'imposait puisqu'il nous fallait travailler à partir d'une distribution formelle de l'autorité. Pour y répondre, nous avons créé des modalités correspondant à la structure du groupe. À la question " la personne est-elle en position théorique de pouvoir? ", nous pouvions donc répondre de l'une des six façons suivantes : "oui, elle est secrétaire "; "oui, elle est vicesecrétaire "; " oui, elle est trésorière "; " oui, elle est chargée de dossier de type 1 »; " oui, elle est chargée de dossier de type 2 »; " non ".

Les deuxième et troisième questions portent sur les interventions. Nous avons d'abord cherché à savoir si, pendant la séance, la personne est intervenue. À la question « la personne se prononce-t-elle? ", nous pouvions répondre de l'une des trois façons suivantes : "oui, à l'aide du langage "; "oui, à l'aide du paralangage "; " non ». La question est incontournable : le pouvoir peut difficilement se mesurer en dehors de l'expression du pouvoir. Certains soutiendront que l'absence d'intervention peut, en 
elle-même, constituer un exercice de pouvoir; mais 1) ils soutiendront, du même coup, presqu'infailliblement, que, de la personne qui s'arroge ainsi le pouvoir, se dégage une certaine arrogance, ou une certaine indifférence... bref, qu'une série de signaux émis de façon non verbale communiquent, en fait, bon nombre d'états d'âmes; et 2) de toute façon, la personne qui est en position de pouvoir ne peut pas rester dans le silence éternellement sans quoi elle ne peut exercer son pouvoir. À la question " quelle est la fréquence à laquelle la personne prend la parole, sous une forme ou une autre? ", nous avons indiqué " sans objet " si nous avions répondu par " non " à la question précédente; sinon, nous avons rapporté le nombre de fois où la personne a pris la parole au cours de la séance étudiée. Pour répondre à la question de la fréquence de la prise de parole, nous avons, bien sûr, tenu compte de chaque fois où un interlocuteur se voyait attribuer ou s'attribuait clairement la parole; mais nous avons aussi choisi de compter comme une nouvelle intervention toute émission de propos qui suivait une interruption de la part d'un autre interlocuteur, que cette émission soit reprise d'idées présentées, suite d'idées en cours ou présentation d'idées nouvelles.

Les quatrième, cinquième, sixième et septième questions portent sur les interruptions. Les deux premières concernent les interruptions initiées par l'interlocuteur. Nous avons répondu par " oui ", par " non " ou par " sans objet " à la question « la personne interrompt-elle les autres ? ». Si nous avions répondu par «non » à cette dernière question, nous répondions par «sans objet " à " combien de fois la personne interrompt-elle les autres? "; sinon, nous dénombrions les interruptions de la part de l'interlocuteur. Les deux autres questions abordent les interruptions dont aurait été victime l'interlocuteur. Ainsi, à la question "la personne se fait-elle interrompre ? ", nous avons répondu par " oui ", par " non " ou par "sans objet "; à la question " quelle est la fréquence à laquelle la personne se fait interrompre par les autres? ", nous avons répondu " sans objet " si nous avions répondu par " non » à la question précédente, sans quoi nous avons indiqué la fréquence à laquelle la personne a été 
interrompue par un autre interlocuteur pendant la réunion, et ce, pour chaque interlocuteur, dans chaque réunion. Pour qu'il y ait interruption, il faut qu'un ou des interlocuteurs définis aient obtenu préalablement le droit de parler. Nous avons qualifié d'interruption toute intervention, interjection, geste ou autre, qui a eu lieu pendant qu'un interlocuteur avait droit de parole, que ce droit lui ait été accordé explicitement — par un détenteur de l'autorité formelle — ou implicitement — par le silence, l'attention, l'écoute des autres. Les conversations parallèles ont été considérées comme des interruptions dans le sens où leurs auteurs ne semblaient pas s'imposer le silence du fait qu'un interlocuteur ait le droit de parole. Au sujet des interruptions : il faut savoir que toute interruption n'est pas affrontement, que l'interruption est aussi souvent ajout, renchérissement, acquiescement, approbation ou encouragement; qu'elle témoigne, en fait, autant de l'aisance ou de la complicité que de l'imposition ou de l'usage de la force symbolique. Il faut aussi savoir que ce qui constitue une interruption, dans l'optique de ce travail, est souvent interruption d'un brouhaha, d'un mélange d'interventions qui nuit au bon fonctionnement de la réunion et, donc, qu'en ces cas, l'interruption est beaucoup plus rappel à l'ordre qu'imposition du soi; si les confusions de propos peuvent être des lieux de création d'ententes, ils peuvent aussi représenter des zones de désaccord, des espaces où sont exprimées des idées contradictoires, où s'entrechoquent des mouvements, où règne la dissonance. Cela étant dit, dans la mesure où l'interruption, quelle que soit sa nature en dehors d'une réaction involontaire, peut appeler l'écoute des autres, et peut, en cela, dans une logique du pouvoir comme détenu, être liée à un pouvoir formel, il nous apparaissait important de tenir compte de sa fréquence.

Les questions huit à onze ont trait aux oppositions. En ce qui les concerne, nous avons d'abord cherché à savoir si les propos d'un interlocuteur avaient fait l'objet d'oppositions : « la personne se bute-t-elle à des oppositions de la part des autres? ". Nous répondions à cette question par " oui ", " non " ou " sans objet". Nous avons, ensuite, cherché à connaître la fréquence de ces 
oppositions : " combien de fois la personne se bute-t-elle à des oppositions? "; nous avons répondu par « sans objet " ou par une valeur absolue. Nous nous sommes, par ailleurs, intéressée aux cas où l'individu était lui-même à la source de l'opposition : « la personne s'oppose-t-elle à des idées émises par d'autres? "; " combien de fois la personne s'oppose-t-elle à des idées émises par d'autres? " Dans le premier cas, nous répondions par " oui ", " non " ou "sans objet "; dans le second, en fonction de la réponse à la question précédente, nous attribuions la valeur « sans objet » ou une valeur absolue. Au cœur de ce questionnement en quatre temps, on trouve l'idée, implicite chez les théoriciens du pouvoir comme détenu, selon laquelle le droit à l'opposition est conférée aux plus grands, voire aux seuls détenteurs d'autorité formelle. Dans notre étude, nous avons mesuré l'opposition de façon verbale, essentiellement; la sémantique a ici été décisive de nos conclusions. Ont été considérées comme des oppositions les interventions consistant à rectifier les propos d'autrui, à apporter une précision ou encore à prendre position quant à ce qui était dit.

Les questions douze à quinze portent, d'une part, sur l'émission d'idées que l'on qualifie de nouvelles en tant qu'elles ne sont pas prévues à l'ordre du jour ou en tant qu'elles naissent de l'échange lui-même; et, d'autre part, sur la reprise, par d'autres, de ces idées. Ainsi, nous nous sommes questionnée de la sorte: " la personne présente-t-elle des idées ou des thèmes qui ne sont pas à l'ordre du jour? »; "la personne présente-t-elle des idées nouvelles? »; " ses idées sont-elles reprises par d'autres?». Chaque fois, nous répondions par "oui ", " non " ou "sans objet ». S’il arrivait que les idées de l'interlocuteur à l'étude fussent reprises par un ou par d'autres individus, alors, à la question " combien de fois ses idées sont-elles reprises par d'autres ? ", nous répondions en indiquant le nombre de fois où il y avait reprise, sinon la question demeurait "sans objet ". L'ordre du jour représente un plan du déroulement de la rencontre dont il fait l'objet; normalement, il est établi en concertation partielle avec les membres de l'organisme, de l'association ou du groupement. On 
peut ainsi le concevoir comme un accord collectif tacite et admettre que son non-respect constitue une infraction que seuls les individus dont le pouvoir le leur confère oseraient commettre. Dans le même esprit, les idées nouvelles étant théoriquement associées à l'autorité, on pourrait s'attendre à ce que seules les personnes qui auraient en principe un ascendant sur le groupe de par leur position se permettent de mettre de l'avant des réflexions nouvelles; ainsi, tout en risquant de déplaire et, donc, de rencontrer l'opposition ou l'opprobre, elles seraient protégées de ces manifestations par leur statut. Les théoriciens du pouvoir comme détenu soutiennent que sa détention - et son maintien, d'ailleurs — sont attribuables aux caractéristiques de l'individu; mais ils reconnaissent également le fait que, par leurs gestes divers, les membres d'une collectivité restreinte viennent consolider les positions des uns et des autres. C'est dans cet esprit que nous avons tenu compte des reprises d'idées nouvelles : si la théorie dit vrai, on devrait s'attendre à découvrir que les idées nouvelles émises par les plus " puissants " soient systématiquement reprises par d'autres; et, à l'inverse, que dans les rares cas où un individu sans titre distinct oserait une telle manœuvre, elle serait sans suivi. Les idées nouvelles, dans le corpus à l'étude, prenaient tantôt la forme d'une suggestion, tantôt la forme d'une interprétation, et leurs reprises, celle d'une reformulation, d'une appropriation ou, encore, d'un non-acquiescement.

Nous avons cherché, avec la seizième question, la trace d'un intérêt particulier : "repère-t-on un fil conducteur, chez la personne, qui témoigne d'un intérêt personnel? " Les modalités, pour cette variable, étaient les suivantes : " oui ", " non ", "sans objet ». Si les théories de l'action disent vrai et que pouvoir et intérêt sont intrinsèquement liés, alors plus la position est imposante, plus l'intérêt personnel devrait être manifeste. Pour repérer l'intérêt, nous avons cherché un fil conducteur, chez l'individu, qui aurait pris la forme d'une trame discursive se développant en marge de la dynamique du groupe.

La dix-septième question s'inscrit dans le net prolongement de la notion d'intérêt telle qu'elle est comprise par ceux pour qui 
l'acteur est au centre de l'analyse : « repère-t-on une stratégie chez la personne? "Si un intérêt est perceptible, c'est que des actions sont entreprises qui consistent à le combler; et, donc, qu'un événement pluritemporel s'observe. À cette question, nous répondions par " oui », «non " ou "sans objet».

Les trois questions suivantes se penchent sur l'harmonie de pensée ou de sentiments : "la personne participe-t-elle à une entente? »; « l'entente est-elle le fruit d'un décret? »; « l'entente est-elle le fruit d'un consensus? " À chacune, nous répondions par " oui ", « non » ou « sans objet ». Les assemblées délibératives ou réunions sont des lieux où s'échangent des idées, où sont émises des positions, où s'expriment des passions, où se font entendre des réserves... Elles sont ainsi un espace dans lequel existent ou prennent forme des accords et des désaccords. Suivant la logique qui fait l'objet de notre critique, on devrait s'attendre à ce que le pouvoir soit déterminant de la capacité à faire agir en fonction de sa volonté; et, donc, que ceux qui en détiennent la plus grande part puissent imposer, par décret, l'adhésion à une idée quelconque, le consensus s'exprimant indépendamment des positions théoriques de pouvoir. Ont été considérées comme des ententes les votes, les répartitions de tâches, l'organisation d'événements et, de façon générale, toute décision collective quant à la structure du groupe.

Les deux questions sur lesquelles se termine l'analyse sont les suivantes : "la personne participe-t-elle à une alliance? ", «l'alliance est-elle le fait de la manipulation de celui ou de ceux qui sont au pouvoir ? " Nous y répondions soit par « oui ", soit par " non ", soit par "sans objet». Une théorie du pouvoir comme détenu soutient qu'un individu peut en faire agir d'autres selon sa volonté du fait du pouvoir qu'il détient; elle suppose aussi que les alliances qui s'expriment, dans des contextes donnés, sont le fruit d'une manipulation à laquelle s'adonnent, encore et toujours, les détenteurs de pouvoir. Ainsi, l'analyse devrait révéler que les alliances, lorsqu'elles surviennent, sont imputables à un individu plutôt qu'à des positions, des affects, des valeurs ou des principes individuels. Nous considérons que plus qu'une simple 
convergence d'idées, l'alliance suppose la durée, de même que l'occultation d'informations qui pourraient amener les "alliés " à transformer leur position et, donc, à rompre l'alliance; et de cela, nous avons tenu compte dans l'analyse.

\section{La description des résultats}

Ont assisté, à chaque réunion, entre 12 et 30 personnes. L'analyse ne tient compte que des personnes qui se prononcent, qu'elles le fassent verbalement, à l'aide du paralangage ou dans une combinaison des deux. En regroupant les cinq réunions, nous obtenons, pour l'analyse, 106 cas, dont 13 cas de personnes qui ne se prononcent pas, 92 de personnes qui se prononcent à l'aide du langage et du paralangage et 1 cas où une personne se prononce à l'aide du paralangage seul; au total, nous comptons donc 93 cas traitables pour l'analyse. Les assemblées réunissent souvent les mêmes individus; certains sont présents à toutes les réunions, d'autres non. Parmi les 93 cas, une même personne peut donc apparaître plus d'une fois. Par souci de cohérence, nous adopterons tout de même les termes "individu " ou " personne ", de façon interchangeable, pour désigner ces cas.

Dans l'ensemble du corpus, le nombre de personnes détenant une position théorique de pouvoir s'élève à 11 .

\subsection{Les fréquences}

Nous avons commencé l'analyse en établissant des fréquences. Dans une séance donnée, le nombre de tours de parole est fort variable : certains individus prennent la parole une seule fois tandis que d'autres la prennent jusqu'à 228 fois. Les interruptions, quant à elles, sont très fréquentes; en fait, elles semblent presque constituer un mode communicationnel : parmi les 93 individus qui se prononcent, 64 interrompent d'autres interlocuteurs et 29 ne le font jamais. Certaines personnes interrompront jusquà 50 fois dans une même séance. Parmi les 93 individus qui se prononcent, 66 sont interrompus - certains jusqu'à 53 fois dans une même séance - et 27 ne le sont pas. Les oppositions, dans les échanges, ne sont pas exceptionnelles : 
27 individus rencontrent des oppositions de la part d'autres membres tandis que 66 n'en rencontrent pas. Dans une même réunion, un individu peut connaître jusquà 7 oppositions à ses propos; à l'inverse, dans une même réunion toujours, une même personne peut s'opposer jusqu'à sept fois à des propos émis par d'autres interlocuteurs. La présentation d'idées ou de thèmes qui ne sont pas à l'ordre du jour est peu fréquente, la chose ne survenant que 11 fois dans l'ensemble des séances. En revanche, des idées nouvelles sont fréquemment présentées. Parmi les 93 personnes à l'étude, on en dénombre 50 qui émettent de telles idées. La reprise de ces idées nouvelles est elle aussi fréquente : 37 individus voient leurs idées reprises par d'autres tandis que 14 individus émettent des idées nouvelles qui ne sont pas reprises. Les idées d'un même individu peuvent être reprises par d'autres jusqu'à huit fois. Il faut souligner que nous avons considéré, dans l'analyse, que le fait de répondre par "voilà " ne constitue pas une reprise au sens où l'on voudrait faire valoir l'idée émise et s'étendre sur elle. Très souvent, le "voilà » sert tout simplement à ponctuer la conversation, à se déplacer vers autre chose. On ne dénote, à aucun moment, un fil conducteur qui témoignerait d'un intérêt personnel et on ne repère aucune stratégie, à proprement parler, chez les interlocuteurs. Les ententes, dans les échanges étudiés, sont fréquentes : parmi les 93 cas à l'étude, il en est 29 qui participent à une entente, 15 qui participent à plus d'une entente et 49 qui ne participent à aucune entente. La question se posait, nous l'avons dit, de savoir si les ententes étaient le fruit d'un décret ou d'un consensus. Les ententes sont, la plupart du temps, le résultat d'un consensus. Dans 38 des 41 cas dans lesquels il y a participation à une entente par consensus, le consensus s'opère entre plusieurs membres du groupes, dont certains qui sont en position théorique de pouvoir et certains qui ne le sont pas; dans les trois autres cas, le consensus est atteint entre des membres qui sont en position théorique de pouvoir, strictement. Nous avons observé, dans l'ensemble des séances, 7 cas où l'individu participait à des ententes par décret. Dans un cas, l'individu participait à une seule entente, à laquelle on 
arrivait par décret, et le décret était celui d'une personne qui était en position théorique de pouvoir. Dans les 6 autres cas, l'individu participait à plus d'une entente. Dans l'un de ces 6 cas, les ententes auxquelles participait l'individu étaient chaque fois le fruit d'un décret de la part de quelqu'un ou de quelques-uns au pouvoir. Dans 4 de ces 6 cas, les ententes étaient parfois le résultat d'un décret de la part de quelqu'un ou de quelques-uns au pouvoir et, dans un cas, les ententes étaient la plupart du temps le résultat d'un décret de la part de quelqu'un ou de quelques-uns au pouvoir. Outre les ententes, nous avons cherché à savoir si des alliances se formaient. Nous avons constaté que les alliances étaient peu nombreuses. La question qui s'imposait ensuite consistait à savoir si ces alliances étaient attribuables à une quelconque manipulation de la part de certains individus, la théorie voulant que les gens au pouvoir soient enclins à former des alliances par lesquelles ils puissent faire prévaloir leurs intérêts personnels. Nous avons observé, au total, 6 alliances; dans aucun cas ces alliances ne sont apparues comme le fait d'une manipulation quelconque. Les personnes se sont ralliées à des positions et des points de vue en énonçant des principes présentés comme étant a priori leurs ou comme ayant été fait leurs par le biais d'idées émises par d'autres lors de la séance, idées par lesquelles elles se voyaient appelées à transformer une position antérieure en fonction de considérations nouvelles, qui étaient telles en tant que soit non connues, soit non appréhendées en amont. Les interventions par lesquelles on a vu des individus - qui étaient ou non en position théorique de pouvoir - se rallier à une position se sont modelées sur des raisonnements qui sont apparus irréductibles à des intérêts personnels, que ces intérêts aient ou non été ceux des plus théoriquement puissants dans le groupe.

Cette première série d'analyses permet de constater : le taux variable de la prise de parole; la récurrence des interruptions; la présence d'oppositions; le faible nombre d'idées qui ne sont pas à l'ordre du jour; le nombre élevé d'idées nouvelles et d'idées reprises; l'apparente absence d'intérêt personnel et de stratégie; la réalisation coutumière d'ententes, qui sont presque toujours le 
fruit d'un consensus; et le faible nombre d'alliances, lesquelles n'apparaissent jamais comme étant le fruit d'une manipulation.

Nous avons procédé à une deuxième série d'analyses, celle-ci corrélationnelle.

\subsection{Les corrélations}

Nous nous sommes d'abord demandée s'il y avait un lien entre la fréquence à laquelle la personne prenait la parole et le nombre de participants assistant à une séance donnée. Nous avons observé que plus il y a de participants, moins chacun tend à prendre le parole, mais le lien entre ces deux variables était faible $^{21}$. Nous nous sommes ensuite demandée s'il y avait un lien entre la fréquence à laquelle la personne prenait la parole et le nombre de fois où elle interrompait les autres. Le lien entre ces deux variables était fort ${ }^{22}$ : plus une personne prend la parole, plus elle interrompt les autres. Nous avons ensuite voulu savoir si la fréquence de prise de parole était, de la même façon, positivement corrélée au nombre d'interruptions subies : nous avons obervé un lien très fort entre les variables, nous révélant que plus une personne prend la parole, plus elle se fait interrompre ${ }^{23}$. Nous nous sommes ensuite questionnée sur le fait de savoir s'il $\mathrm{y}$ avait un lien entre le nombre de fois où la personne se butait à des oppositions de la part des autres et le nombre de fois où elle se faisait interrompre. Le lien entre les variables s'est avéré positif et assez fort ${ }^{24}$ : plus une personne se bute à des oppositions, plus elle se fait interrompre. Est-ce que, de la même façon, plus une personne se bute à des oppositions, plus elle interrompt les autres? Telle était notre prochaine question; la réponse : oui, dans une grande mesure ${ }^{25}$. Nous avons cherché à savoir s'il y avait un lien entre le nombre de fois où la personne s'opposait à des idées émises par d'autres et le nombre de fois où elle se faisait interrompre et avons découvert que le lien entre ces variables était

\begin{tabular}{ll}
\hline 21 & $\mathrm{r}=-0,20 ; \mathrm{p}<0,05$. \\
22 & $\mathrm{r}=0,93 ; \mathrm{p}<0,001$. \\
23 & $\mathrm{r}=0,92 ; \mathrm{p}<0,001$. \\
24 & $\mathrm{r}=0,64 ; \mathrm{p}<0,001$. \\
25 & $\mathrm{r}=0,59 ; \mathrm{p}<0,001$.
\end{tabular}


fort $^{26}$ : plus, en effet, une personne s'oppose aux idées des autres, plus elle se fait interrompre. De la même façon, une autre analyse nous a révélé que plus une personne s'oppose à des idées émises par d'autres, plus elle interrompt les autres; le lien était encore assez fort entre les deux variables ${ }^{27}$. Y a-t-il un lien entre le nombre de fois où les idées de la personne sont reprises par d'autres et le nombre de fois où elle se fait interrompre ? Entre le nombre de fois où les idées de la personne sont reprises par d'autres et le nombre de fois où elle interrompt les autres ? À ces deux questions, nous avons répondu par l'affirmative : plus les idées de l'individu sont reprises, plus l'individu se fait interrompre $^{28}$ et plus les idées de l'individu sont reprises, plus l'individu interrompt les autres ${ }^{29}$.

Les analyses corrélationnelles révèlent donc que plus une personne prend la parole, plus elle interrompt les autres et se fait interrompre; que plus elle se fait interrompre et interrompt les autres, plus elle se bute à des oppositions et présente des oppositions; et que plus ses idées sont reprises, plus elle se fait interrompre et interrompt les autres. Autrement dit, plus l'individu prend la parole, plus il advient de phénomènes - interruptions, oppositions, etc. — liés à son activité communicationnelle. Cela peut paraître évident; mais rappelons que 1) le travail du sociologue consiste précisément à transformer en hypothèses les énoncés du sens commun; 2) cela ne relève certainement pas de l'évidence pour les détenteurs des théories de l'action dont les postulats appellent des conclusions qui vont exactement dans le sens contraire de nos observations. La question se pose alors de savoir si la prise de parole et, donc, les interruptions, les oppositions, l'émission et la reprise d'idées varient selon la position de l'individu. Pour le vérifier, nous avons procédé à une analyse de variance à un facteur, donc à un troisième registre d'analyses.

\begin{tabular}{ll}
\hline 26 & $\mathrm{r}=0,73 ; \mathrm{p}<0,001$. \\
27 & $\mathrm{r}=0,65 ; \mathrm{p}<0,001$. \\
28 & $\mathrm{r}=0,62 ; \mathrm{p}<0,001$. \\
29 & $\mathrm{r}=0,61 ; \mathrm{p}<0,001$.
\end{tabular}




\subsection{Les anova}

Les analyses ont révélé une différence de moyennes pour les variables suivantes : fréquence à laquelle la personne prend la parole $^{30}$; nombre de fois où la personne interrompt les autres ${ }^{31}$; fréquence à laquelle la personne se fait interrompre par les autres $^{32}$; nombre de fois où la personne se bute à des oppositions $^{33}$; nombre de fois où la personne s'oppose à des idées émises par d'autres ${ }^{34}$; nombre de fois où ses idées sont reprises par d'autres ${ }^{35}$. Des tests post hoc ${ }^{36}$ révèlent que le secrétaire du groupe se démarque toujours radicalement des autres membres de l'assemblée. Par séance, il prend la parole, en moyenne, 189,6 fois; il interrompt les autres, en moyenne, 31,4 fois; il se fait interrompre, en moyenne, 38,4 fois; il se bute à des oppositions, en moyenne, 3,8 fois; il s'oppose à d'autres, en moyenne, 4,8 fois; ses idées sont reprises, en moyenne, 4,4 fois. Les tests post hoc révèlent, de même, que les chargés de dossiers de type 1 se démarquent toujours des autres, tandis que les autres, entre eux, ne se démarquent pas.

\section{Linterprétation des résultats}

Nous avons débuté l'analyse en établissant des fréquences. Cet exercice a permis d'observer que certains prennent plus souvent la parole que d'autres, que les interruptions sont très fréquentes et qu'il n'est pas rare pour un individu de s'opposer aux idées d'un autre, comme il n'est pas rare que l'on s'oppose aux siennes. Il a, de même, mis en évidence le fait que si l'on respecte normalement l'ordre du jour, cela n'empêche pas que des propos qui circulent naissent des idées nouvelles, inattendues, qui sont bien souvent reprises par d'autres. Il a donc permis de constater que

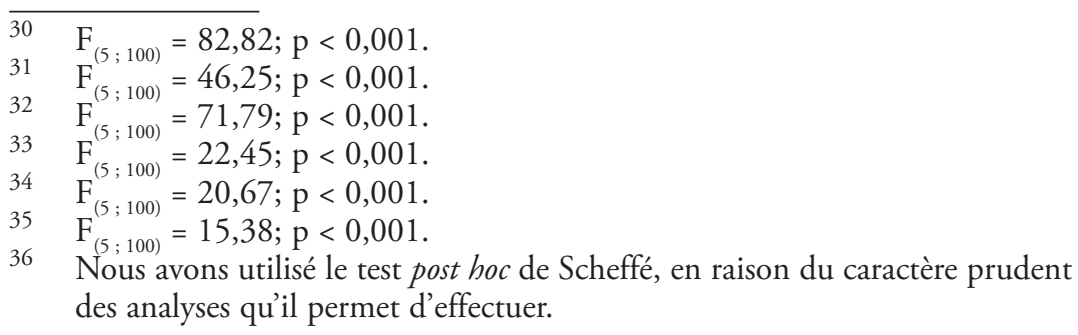


les échanges sont ontologiquement dynamiques et que, s'ils s'inscrivent dans un cadre prédéfini, ce cadre ne contient pour autant la totalité de ce qui peut advenir, de ce qui peut émerger ou résulter de l'échange, lequel comporte toujours une part d'imprévu. Plus encore, l'exercice a permis de conclure à l'absence d'intérêt personnel et de stratégie, du moins, d'un intérêt ou d'une stratégie qui auraient été apparents. Et dans la mesure où l'intérêt et la stratégie inconscients constituent des non-sens en ce que l'un et l'autre ont pour condition d'existence préalable la conscience $^{37}$, leur non-révélation apparaît comme une condition suffisante pour déclarer leur non-existence. Dans une logique du pouvoir comme détenu, donc dans les théories de l'action, par exemple, le pouvoir est entre autres pouvoir de faire agir en fonction de son intérêt en élaborant des stratégies à cet effet; force est de constater que l'analyse donne ici tort à ce principe, principe qui est d'autant plus réfutable que l'exercice, a, de plus, révélé que les ententes sont presque toujours le fruit d'un consensus et non celui d'un décret et que les alliances n'apparaissent jamais comme étant le résultat d'une manipulation. Déjà, à s'en tenir aux simples fréquences, le pouvoir apparaît peu en mesure de rendre compte de ce qui advient dans les échanges observés. Poursuivons tout de même en dégageant, des corrélations et des analyses de variance effectuées, les constats qui s'imposent.

Les corrélations ont fait valoir l'importance de la prise de parole et nous ont amenée à poser la question de la différence de moyennes selon la position.

Les analyses de variance à un facteur et les tests post hoc qui les accompagnent ont révélé des différences de moyennes radicalement plus élevées chez le secrétaire du groupe que chez les autres membres. Il se démarque nettement de tous les autres en ce qu'il prend la parole, interrompt les autres et s'oppose à d'autres beaucoup plus souvent que quiconque. Ses idées sont aussi plus souvent reprises que ne le sont celles des autres. De la même façon, les chargés de dossiers de type 1 se démarquent du reste

37 Voir Mélanie Girard, Contribution à la critique des théories de l'action, op. cit., p. 89. 
du groupe alors que le reste du groupe est essentiellement homogène. Mais le secrétaire, puis les chargés de dossiers de type 1 dans une mesure moindre, sont aussi ceux qui se font interrompre le plus souvent et ceux à qui l'on s'oppose le plus souvent. Si l'on peut soutenir que la prise de parole, la tendance à interrompre et à s'opposer et la reprise de ses idées sont, en théorie, tributaires du pouvoir, on ne peut, à l'inverse, soutenir que le fait de se faire interrompre ou que l'on s'oppose à soi témoignent de la détention du pouvoir. On pourrait même dire, à l'opposé, que ce sont là des signes - théoriques toujours - de non-pouvoir.

Les personnes qui sont en position théorique de pouvoir sont souvent celles qui se prononcent le plus souvent; cela est particulièrement vrai du secrétaire et des chargés de dossier de type 1. Ces personnes détiennent des informations privilégiées du fait de leur position, informations qu'elles sont tenues de partager avec le groupe; en cela, il n'est pas étonnant de les voir se prononcer, en moyenne, plus souvent que les autres. Les échanges mettent donc beaucoup en relation des individus dont la marge théorique de pouvoir est la plus grande; cependant, ils mettent également en lien avec les individus dont la marge de pouvoir est la plus grande ceux dont la marge est moindre, et ce, dans une logique qui rend possible que les seconds interrompent les premiers ou, encore, qu'ils s'opposent à eux. Plus encore, s'il est vrai que secrétaire et chargés de dossiers de type 1 se démarquent des autres, les autres, entre eux, ne se démarquent point. Or, il faut souligner que figurent, parmi «les autres ", des chargés de dossier de type 2 et le vice-secrétaire, donc des individus qui sont en position théorique de pouvoir. Le fait que l'on n'observe aucune différence entre eux et ceux qui ne détiennent aucune position théorique de pouvoir constitue une autre invitation à repenser la place du pouvoir comme catégorie analytiquement utile dans l'analyse des relations humaines.

Ajoutons par ailleurs que si l'on reprend plus souvent les idées du secrétaire du groupe que celles des autres, on le fait bien souvent parce que son intervention traite d'un point qui est à l'ordre du jour et qu'on est appelé à se prononcer, en tant que 
chargé de dossier de type 1 ou 2, sur ce point. Ainsi, la reprise de l'idée du secrétaire est alors bien simplement une élaboration d'un point à l'ordre du jour qui transite par le secrétaire. Cela étant, en ayant à l'esprit que le pouvoir est théoriquement lié à l'intérêt personnel; et que l'intérêt personnel, dans une distribution formelle de l'autorité, est théoriquement lié à la position de l'individu, il faut dire qu'il serait malhabile de conclure à la manifestation d'un intérêt personnel dans la plupart des idées que véhicule le secrétaire, idées qui sont, plus souvent que celles des autres, reprises par d'autres membres du groupe.

\section{La conclusion}

Il existe, en sciences sociales, une conception selon laquelle le pouvoir est déterminant de l'action, et donc des relations humaines. Chez les adeptes de cette vision, le pouvoir est fonction de la position sociale et il a pour corollaire l'intérêt : meilleure est la position sociale de l'individu, plus est grande sa marge de pouvoir et plus il est à même de faire agir les autres en fonction de son intérêt. Le pouvoir existe donc en dehors de l'individu, en lui-même, en tant qu'il est déterminé par une position qui est, elle, objectivée; et il est soumis à la contextualité. Mais il existe aussi, en sciences sociales, une autre conception du pouvoir qui, elle, le pose comme catégorie non analytiquement utile pour comprendre les relations humaines : elle insiste sur l'importance de la socialité, de l'historicité et de l'émoraison. Des travaux nous ont amenée à privilégier la seconde de ces visions en ce qu'ils ont fait valoir la place marginale de l'intérêt, de l'intention et de la stratégie dans les relations humaines en démontrant que ce qui advient en elles n'est réductible ni à l'intérêt, ni à l'intention, ni à la stratégie; et en faisant valoir que l'intérêt, l'intention et la stratégie transitent, de toute façon, par les relations humaines qui, elles, supposent la socialité, l'historicité et l'émoraison. Mais ces travaux laissaient en plan une critique : ils ne permettaient pas l'étude des structures de pouvoir formel dans le temps. Il nous a donc semblé qu'un travail qui consisterait en une vérification de la valeur heuristique de la catégorie "pouvoir ", 
modélisée en fonction d'une distribution formelle de l'autorité, s'imposait; c'est à cette tâche que nous nous sommes consacrée. Nous l'avons fait en nous dotant d'indicateurs du pouvoir tel que le conceptualisent les théories qui le perçoivent comme détenu et en soumettant la grille d'analyse ainsi conçue à l'étude de relations, observées sur une période de huit mois, au sein d'un groupe affichant une distribution formelle de l'autorité.

Les données obtenues ont été soumises à une analyse en trois temps, qui a permis de conclure, d'abord, à l'absence d'intérêt personnel ou de stratégie, à la dominance des ententes par consensus et à l'absence d'alliances par manipulation. L'analyse a révélé, ensuite, que plus on prend la parole, dans un groupe donné, plus on interrompt les autres, plus on s'oppose aux idées émises, mais aussi, plus on se fait interrompre et plus on rencontre d'oppositions de la part des autres. Elle a aussi montré que, si certains des individus dont l'autorité formelle est la plus imposante sont ceux qui se prononcent le plus et, donc, qui interrompent et s'opposent le plus souvent aux propos d'autrui, ce sont aussi ceux qui se font interrompre et à qui l'on s'oppose le plus souvent, les interruptions provenant à la fois de gens détenant une position de pouvoir et de gens n'en détenant pas. Elle a, enfin, fait valoir l'importance de la non-distinction entre individus dotés de pouvoir théorique et individus non dotés de pouvoir théorique. Et tout cela a permis de conclure à l'absence d'effet du pouvoir théorique tel qu'il a été modélisé ici, en fonction des postulats que l'on tire des théories dans lesquelles le pouvoir apparaît comme chose détenue.

Le pouvoir, en tant que catégorie analytique issue de l'autorité formelle, apparaît ici peu utile : il ne donne cours à la perceptibilité ni d'un intérêt ni d'une stratégie; il ne détermine pas les ententes ou les alliances qui s'expriment à travers les relations; il n'empêche pas que l'on s'oppose à soi ou que l'on se fasse interrompre; il ne permet même pas, pour certains postes, que les individus qui en détiennent se distinguent de ceux qui n'en détiennent pas. 
S'il est vrai que les personnes qui se prononcent le plus souvent sont en position théorique de pouvoir, il ne faut pas oublier que les positions de pouvoir sont liées à des mandats, lesquels obligent les personnes mandatées à se rapporter au groupe. En cela, elles sont moins liées à un pouvoir de faire agir quà une obligation de rapporter. Et en rapportant, ces personnes s'exposent à des interruptions et à des oppositions, lesquelles surviennent parce que les idées qu'elles véhiculent suscitent, chez ceux qui y sont exposés, des réactions qui sont beaucoup plus le fait de positions individuelles que d'une position théorique de pouvoir. Pouvoir formel et pouvoir effectif semblent bien peu liés : des propos sont véhiculés, bien souvent par une personne qui doit les rapporter au groupe et qui, de ce fait, est en position théorique de pouvoir; les individus, quelle que soit leur position, réagissent à ces propos en faisant valoir des oppositions, des accords, en demandant des précisions; ces oppositions, ces accords, ces demandes de précisions expriment un rapport à l'information qui est forcément émorationnel plutôt que rationnel et qui se dessine à travers la socialité qui inscrit l'individu dans le groupe et dans la collectivité en général et à travers l'historicité qui caractérise le rapport de l'individu au groupe, qui rend possible l'expression elle-même. Et ces oppositions, ces accords, ces précisions s'inscrivent dans des discussions, donc dans des dynamiques qui se construisent sur des préoccupations communes, sur des intérêts partagés, émotivement, rationnellement, sur des questions qui sont davantage liées aux thématiques que l'on peut associer à la raison d'être du groupe, à sa cause, si l'on peut parler ainsi, qu'elles ne se rattachent à l'intérêt personnel de tel ou de tel autre membre détenteur d'une forme quelconque de pouvoir et qui chercherait, dans l'ensemble de ses interactions, à consolider ce pouvoir. Le pouvoir apparaît donc beaucoup plus relationnel que déterminé par la distribution formelle de l'autorité; et dans la mesure où il est un produit de la relation, il ne saurait à lui seul, s'imposer en extériorité, comme une catégorie analytique qui suffirait à rendre compte de la circulation de l'information.

Cet exercice d'opérationnalisation peut apparaître étonnant. 
Il est vrai que la notion de pouvoir n'est pas habituellement opérationnalisée sinon de façon indirecte à partir notamment des notions de classe sociale, de prestige, de salaire ou de statut. Mais cela n'élimine en rien la démarche que voici, qui constitue non pas une critique absolue de la notion de pouvoir, mais bien une contribution empirique, faite à partir d'indicateurs qui, s'ils ne sont pas exclusifs, permettent néanmoins de constater que le pouvoir formel ne suffit pas à expliquer tout ce que rendent possible les dynamiques humaines.

\section{Bibliographie}

Bagaoui, Rachid, «Un Paradigme systémique relationnel est-il possible? Proposition d'une typologie relationnelle ", Nouvelles perspectives en sciences sociales, vol. 3, $\mathrm{n}^{\circ}$ 1, 2007, p. 151-175.

Bouchard, Pierre, "La Théorie de l'action et les parcours de vie ", Nouvelles perspectives en sciences sociales, vol. 1, no 2, 2006, p. 67-114.

Caillé, Alain, Critique de la raison utilitaire, Paris, La Découverte, 1989.

Caillé, Alain et Jacques T. Godbout, L'Esprit du don, Paris, La Découverte, coll. "Textes à l'appui / Textes anthropologiques ", 1992.

Crozier, Michel et Erhard Friedberg, L'Acteur et le système : les contraintes de l'action collective, Paris, Seuil, coll. "Sociologie politique ", 1981 [1977].

Foucault, Michel, Histoire de la sexualité I. La volonté de savoir, Paris, Gallimard, coll. «Tel », 1994 [1976].

Friedberg, Erhard, Le Pouvoir et la Règle, Paris, Seuil, coll. "Sociologie ", 1993.

Girard, Mélanie, Relations humaines et production d'information : l'échange comme objet d'étude d'une approche relationnelle, mémoire de maîtrise, Sudbury, Université Laurentienne, 2004.

Girard, Mélanie, "Éléments de critique des théories de l'action ", Nouvelles perspectives en sciences sociales, vol. $3, \mathrm{n}^{\circ} 1,2007$, p. 47-60.

Girard, Mélanie, Contribution à la critique des théories de l'action. Intention et émoraison, thèse de doctorat, Toulouse, Université des Sciences Sociales de Toulouse I, 2009. 
Girard, Mélanie, Simon Laflamme et Pascal Roggero, "L'Intention est-elle si universelle que ne le prétendent les théories de l'action ? ", Nouvelles perspectives en sciences sociales, vol. 1, $\mathrm{n}^{\circ} 2,2006, \mathrm{p} .115-148$.

Habermas, Jürgen, Théorie de l'agir communicationnel, tomes I et II, Paris, Fayard, coll. "L'espace du politique », 1987 [1981].

Hegel, Georg Wilhelm Friedrich, La Phénoménologie de l'esprit, Paris, Gallimard, coll. "Bibliothèque de philosophie ", 1993 [1807].

Jalbert, Paul, "Analyse du rôle de l'intention dans les échanges dyadiques ", Nouvelles perspectives en sciences sociales, vol. 2, n ${ }^{\circ}$ 1, 2006, p. 101-141.

Ladrière, Paul, Patrick Pharo et Louis Quéré, La Théorie de l'action. Le sujet pratique en débat, Paris, CNRS, 1993.

Laflamme, Simon, La Société intégrée. De la circulation des biens, des idées et des personnes, New York / Bern / Paris, Peter Lang, 1992.

Laflamme, Simon, Communication et émotion. Un essai de microsociologie relationnelle, Paris, L'Harmattan, coll. "Logiques sociales », 1995.

Laflamme, Simon, Suites sociologiques, Sudbury, Prise de parole, coll. "Épistémè », 2006.

Machiavel, Nicolas, Le Prince, traduit et commenté par Jean-Louis Fournel et Jean-Claude Zancarini, texte italien établi par Giorgio Inglese, Paris, Presses universitaires de France, coll. "Fondement de la politique ", 2000 [1532].

Morin, Edgar, Introduction à la pensée complexe, Paris, ESF, 1992.

Roggero, Pascal, De la complexité des politiques locales, Paris, L'Harmattan, 2005.

Vautier, Claude, Le Repli et l'imagination. Essai de modélisation complexe des phénomènes territoriaux : le cas de l'intercommunalité dans le département $d u$ Tarn, thèse de doctorat, Toulouse, Université des Sciences Sociales de Toulouse I, 2005.

Vautier, Claude, "Essai de modélisation complexe en sciences sociales : le cas de l'intercommunalité dans le département du Tarn ", Nouvelles Perspectives en Sciences Sociales, vol. 1, n 2, 2006, p. 149-217.

Vautier, Claude, "La Longue marche de la sociologie relationnelle", Nouvelles perspectives en sciences sociales, vol. 4, no 1, 2008, p. 77-106.

von Foerster, Heinz, Observing Systems: Selected Papers of Heinz von Foerster, Seaside (CA), Intersystems Publications, 1981.

Weber, Max, Économie et société, Paris, Plon, coll. « Recherches en sciences humaines ", 1971 [1922]. 